\title{
Assessing the Influence of Quality Improvement Methods to Improve the Success Rate of Peripheral Venous Indwelling Needle
}

\author{
Yanhua Shi ${ }^{1}$, Xiaolan Wang ${ }^{1}$, , Quan Jiang ${ }^{1}$, Weirong $\mathrm{Li}^{1}$, Huiling Liu ${ }^{2}$, Mengying $\mathbf{Q i}^{3}$ \\ ${ }^{1}$ Hepatological Surgery Department, The First Affiliated Hospital of Jinan University, Guangzhou, China \\ ${ }^{2}$ Kidney Surgery and Thoracic Surgery of Department, The First Affiliated Hospital of Jinan University, Guangzhou, China \\ ${ }^{3}$ College of Nursing, Jinan University, Guangzhou, China \\ Email address: \\ 59235310@qq.com (Yanhua Shi), wangxiaolan74@126.com (Xiaolan Wang), 151733060@qq.com (Quan Jiang), \\ 674656359@qq.com (Huiling Liu),2827758463@qq.com (Weirong Li),347039102@qq.com (Mengying Qi) \\ ${ }^{*}$ Corresponding author
}

\section{To cite this article:}

Yanhua Shi, Xiaolan Wang, Quan Jiang, Weirong Li, Huiling Liu, Mengying Qi. Assessing the Influence of Quality Improvement Methods to Improve the Success Rate of Peripheral Venous Indwelling Needle. American Journal of Nursing Science. Vol. 9, No. 4, 2020 , pp. $190-192$. doi: 10.11648/j.ajns.20200904.12

Received: April 27, 2020; Accepted: May 25, 2020; Published: June 3, 2020

\begin{abstract}
Objective: To assess the influence of Quality improvement methods to improve the success rate of peripheral venous indwelling needle. Methods: 1613 patients were invested to join our study, they provided 2472 cases of peripheral venous indwelling needle. The time of they received services is from March 2019 to December 2019. They were divided into a control group (participant: $n=595$, case: $n=1035$ ) and an intervention group (participant: $n=1018$, case: $n=1437$ ) according to this time, that patients were invested to join control group who receive services between March 2019 and June 2019, intervention group participants' service time is between June 2019 and December 2019. The control group participants receive routine care model in the PIVC process. About collection, the information includes the success rate of PIVC, complications situation and indwelling time of PIVC. Result: The success rate of PIVC assessment from researcher's report, they record the result of patient's PIVC. Intervention group has higher success rate than that of control group (71.95\% vs 49.57\%). Most complications were happened in PIVC process. it shows the research result of mainly 4 kinds of complication in the table, the result contains seepage, blocking pipe, take off the tube and phlebitis. In overall, intervention group patients have less complications cases and lower incidence of complications in each kind of complication, except as phlebitis result ( $\mathrm{p}$ $=0.369$ ). Conclusion: the quality improvement methods can improve the success rate of PIVC and reduce the complications cases of PIVC.
\end{abstract}

Keywords: PIVC, Quality Improvement, Peripheral Venous Indwelling Needle

\section{Introduction}

Insertion of a peripheral intravenous cannula (PIVC) is one of the most common procedures performed in the emergency department (ED). According to Australian hospital's report, the hospital used approximately 25 million PIVC each year, and two billion used globally [1,2]. Its function included providing patients with rapid intravenous fluids, life-saving medications and blood transfusions, PIVC are an invaluable clinical tool [3]. In recent research, however, between $18 \%$ and $50 \%$ of all PIVCs inserted were remain unused in ED
[4-6]. However, the PIVC still had some benign and risk. Insertion of PIVC can be painful for the patient, and indwelling PIVC can cause for complications, such as occlusion, infiltration, phlebitis and infection [7, 8]. The staphylococcus aureus bacteraemia is one of frequent serious complication of the PIVC, PIVC-related staphylococcus aureus bacteraemia represent as much as $25 \%$ of all health care-associated staphylococcus aureus bacteraemia episodes $[9,10]$. In addition, even worse situation is multiple attempts, it is common in PIVC insertion. There is exacerbate negative experiences of patients [11]. 
Healthcare quality is defined as that the application of medical science and technology in a manner that maximizes its benefit to health without correspondingly increasing the risk. In other define, the healthcare quality is provision of care that exceeds patient expectations and achieves the highest possible clinical outcomes with the resources available $[12,13]$. One of important components of quality health care is nursing care [14]. In fact, nurses play important roles, such as regulating quality care and improving health care values [15]. However, quality of nursing care can be affected by many factors of hospital, that include resource limitations, nursing documentation, the hospital nursing system, knowledge, skill and attitude, and communication with other care takers, patients and their families [16]. Aim of this study is that assess the influence of quality improvement methods to improve the success rate of peripheral venous indwelling needle.

\section{Methods}

\subsection{Participants Enrollment and Survey Methods}

1613 patients were invested to join our study, they provided 2472 cases of peripheral venous indwelling needle. The time of they received services is from March 2019 to December 2019. They were divided into a control group (participant: $n=$ 595, case: $n=1035$ ) and an intervention group (participant: $n$ $=1018$, case: $n=1437$ ) according to this time, that patients were invested to join control group who receive services between March 2019 and June 2019, intervention group participants' service time is between June 2019 and December 2019. The control group participants receive routine care model in the PIVC process. Additionally, we use nursing model of quality improvement methods. Our researchers collect the information by hospital recoding, questionnaire and interview in PIVC process, the information include the success rate of PIVC, complications situation and indwelling time of PIVC.

Their inclusion criteria were: (1) The patients undergoing peripheral venous indwelling needle; (2) They had good mental health; (3) Patients volunteered to participate in post-treatment follow-up. Their withdraw criteria were: (1) surgical patients received open indwelling needle in the operating room; (2) Patients with short - term peripheral venous hypernutrition.

\subsection{Statistical Analysis}

Survey responses were analyzed using descriptive statistics. Sample proportions, means, and standard deviation (SD) are reported. All analyses were performed in SPSS 24.

\section{Result}

The Table 1 shows patient characteristics of control group and intervention group, their gender data and age data are similar in our study. But the indwelling time of PIVC is different between group, intervention group patients have longer indwelling time of PIVC than that of control group, and the result is statistical significance $(\mathrm{p}=0.041)$.

Table 1. Patient Characteristics (Mean $\pm S D$ ).

\begin{tabular}{llll}
\hline Projects & Gender (female), n(\%) & Age (year) & Indwelling time of PIVC (hour) \\
\hline Intervention group $(\mathrm{n}=1437)$ & $712(49.5 \%)$ & $37.44 \pm 17.17$ & $70.22+48.33$ \\
Control group $(\mathrm{n}=1035)$ & $551(53.2 \%)$ & $38.81 \pm 19.21$ & $60.59+41.90$ \\
$\mathrm{t}$ & 0.314 & 1.613 & 3.198 \\
$\mathrm{P}$ value & 0.913 & 0.605 & 0.041 \\
\hline
\end{tabular}

The success rate of PIVC assessment from researcher's report, they record the result of patient's PIVC (Table 2).

Intervention group has higher success rate than that of control group ( $71.95 \%$ vs $49.57 \%)$, it is statistical significance.

Table 2. Success rate of PIVC.

\begin{tabular}{lll}
\hline Projects & Success case (n) & Percent (\%) \\
\hline Intervention group $(\mathrm{n}=1437)$ & 1034 & $71.95 \%$ \\
Control group $(\mathrm{n}=1035)$ & 513 & $49.57 \%$ \\
P value & $<0.001$ & $<0.001$ \\
\hline
\end{tabular}

Most complications were happened in PIVC process. The Table 3 shows the research result of mainly 4 kinds of complication in the table, the result contains seepage, blocking pipe, take off the tube and phlebitis. In overall, intervention group patients have less complications cases and lower incidence of complications in each kind of complication, except as phlebitis result $(\mathrm{p}=0.369)$.

Table 3. Complications situation of PIVC [n(\%)].

\begin{tabular}{lllll}
\hline Projects & Seepage & Blocking pipe & Take off the tube & Phlebitis \\
\hline Intervention group $(\mathrm{n}=1437)$ & $185(12.9 \%)$ & $18(1.3 \%)$ & $19(1.3 \%)$ & $181(12.6 \%)$ \\
Control group $(\mathrm{n}=1035)$ & $311(30.0 \%)$ & $42(4.1 \%)$ & $25(2.4 \%)$ & $118(11.4 \%)$ \\
$\mathrm{X}^{2}$ & 110.645 & 19.994 & 4.113 & 0.808 \\
$\mathrm{P}$ value & $<0.005$ & $<0.005$ & 0.043 & 0.369 \\
\hline
\end{tabular}




\section{Discussion and Conclusion}

Base on some report, the vessel walls can be damaged and scared by PIVC. There is increasing evidence that clinicians should act to preserve the integrity of patients' vessels [17]. After inserting a PIVC without clinical indication, if it was leaved or idled, the patients are going to meet avoidable harms and risks [18]. Anecdotal evidence suggests this maybe common in EDs however, accurate assessment of the insertion and use of PIVCs in ED is hard to establish. In addition, there are many complications associated with PIVCs in pediatric patients, such as infiltration, embolism, and phlebitis. Unfortunately, incidence of phlebitis among pediatric patients ranges from 1.5 to $71 \%$ [19]. However, the incidence of phlebitis increases in the second and third days of the PIVC insertion [20].

Base on our research result, the quality improvement methods can improve the success rate of PIVC and reduce the complications cases of PIVC. In success rate research of PIVC, the intervention group has higher success rate in treatment process, it indicates that the quality improvement methods have strongly influence to PIVC in success rate. For the complication situation of PIVC, it contains 4 main domains of complications, such as seepage, blocking pipe, take off the tube, and phlebitis. The quality improvement methods have better improvement to seepage, blocking pipe, and take off the tube, but it has week influence to phlebitis domain. Thus, the quality improvement methods can reduce the complications rate of PIVC.

\section{References}

[1] Alexandrou E, Ray-Barruel G, Carr PJ, Frost SA, Inwood S, Higgins N, et al. Useof short peripheral intravenous catheters: characteristics, management, and outcomes worldwide. J Hosp Med 2018; 13 (5).

[2] Australian Institute of Health and Welfare. Australian hospital statistics 2017-2018. Canberra: AIHW; 2018. Available from: https://www.myhospitals.gov.au/hospital/310000011/princessalexandra-hospital/emergency-department.

[3] Carr PJ, Higgins NS, Cooke ML, Mihala G, Rickard CM. Vascular access specialist teams for device insertion and prevention of failure. Cochrane Database Syst Rev. 2018; 3: CD011429.

[4] Hawkins T, Greenslade JH, Suna J, Williams J, Rickard CM, Jensen $\mathrm{M}$, et al. Peripheral intravenous cannula insertion and use in the emergency department: an intervention study. Acad Emerg Med 2018; 25 (1): 26-32.

[5] Fry M, Romero B, Berry A. Utility of peripheral intravenous cannulae inserted in one tertiary referral emergency department: a medical record audit. Australas Emerg Nurs J. 2016; 19 (1): 20-25.
[6] Decker K, Ireland S, O'Sullivan L, Boucher S, Kite L, Rhodes $\mathrm{D}$, et al. Peripheral intravenous catheter insertion in the emergency department. Australas Emerg Nurs J. 2015; 19: $138-144$.

[7] Marsh N, Webster J, Larson E, Cooke M, Mihala G, Rickard CM. Observational study of peripheral intravenous catheter outcomes in adult hospitalized patients: a multivariable analysis of peripheral intravenous catheter failure. J Hosp Med 2018; 13 (2): 83-89.

[8] Mermel LA. Short-term peripheral venous catheter-related bloodstream infections: a systematic review. Clin Infect Dis 2017; 65 (10): 1757-1762.

[9] Austin ED, Sullivan SB, Whittier S, Lowy FD, Uhlemann AC. Peripheral intravenous catheter placement is an underrecognized source of Staphylococcus aureus bloodstream infection. Open Forum Infect Dis. 2016; 3 (2): 72.

[10] Stuart RL, Cameron DR, Scott C, Kotsanas D, Grayson ML, Korman TM, et al. Peripheral intravenous catheter-associated staphylococcus aureus bacteraemia: more than 5 years of prospective data from two tertiary health services. Med J Aust. 2013; 198 (10): 551-553.

[11] Becerra MB, Shirley D, Safdar N. Prevalence, risk factors, and outcomes of idle intravenous catheters: an integrative review. Am J Infect Control. 2016; 44 (10): 167-172.

[12] Donabedian A. Michigan, \& Health Administration Press. The definition of quality and approaches to its assessment. Ann Arbor. 1980.

[13] Ovretveit J. Blackwell. Health service quality: An introduction to quality methods for health services. Oxford. 1992.

[14] Ali MM. A conceptual framework for quality of care. Materia Socio Medica. 2016; 24 (4): 251-326.

[15] Leslie WH, et al. Quality and nursing: Moving from a concept to a core competency. Urologic Nursing. 2018; 28 (6): 417-426.

[16] The Role of Nurses in Hospital Quality Improvement. Center for Studying Health System Change Research Brief No. 3. 2018. http://hschange.org/CONTENT/972/index.html.

[17] Moureau N. In: Moureau N, editor. Vessel health and preservation: the right approach for vascular access. Basel, Switzerland: Springer Nature; 2019.

[18] Gledstone-Brown L, McHugh D. Review article: Idle 'just-in-case' peripheral intravenous cannulas in the emergency department: is something wrong? Emerg Med Australas. 2018; 30 (3): 309-312.

[19] Bitencourt ES, Leal CN, Boostel R, Mazza V, Felix JVC, Pedrolo E. Prevalence of phlebitis related to the use of peripheral intravenous devices in children. Cogitare Enferm. 2018; 23 (1): e49361.

[20] Webster J, Osborne S, Rickard CM, New K. Clinically-indicated replacement versus routine replacement of peripheral venous catheters. Cochrane Database of Systematic Reviews. 2015: 8. 